\title{
Regional Eigenvalue-Clustering Robustness Analysis for Linear Discrete Time-Delay Systems with Both Structured and Unstructured Parameter Uncertainties*
}

\author{
Shinn-Horng $\mathrm{CHEN}^{* *}$ and Jyh-Horng $\mathrm{CHOU}^{* * *}$
}

\begin{abstract}
In this paper, the problem of the regional eigenvalue-clustering robustness analysis for linear discrete time-delay systems with both structured (elemental) and unstructured (normbounded) parameter uncertainties is investigated. Under the assumption that all the eigenvalues of a linear nominal discrete time-delay system lie within a specified region, a new sufficient condition is proposed to preserve the assumed property when both the structured (elemental) and the unstructured (norm-bounded) parameter uncertainties are added into the linear nominal discrete time-delay system. No restriction is imposed on the shapes of the specified region. When all the eigenvalues are just required to locate inside the unit circle, the proposed criterion will become the robust stability criterion. For the case that the linear discrete time-delay system only subjects to structured (elemental) parameter uncertainties, by two illustrative examples, the presented sufficient condition is shown to be less conservative than the existing one reported recently in the literature.
\end{abstract}

Key Words: Regional Eigenvalue-Clustering Robustness, Discrete Time-Delay Systems, Structured and Unstructured Parameter Uncertainties

\section{Introduction}

To ensure both stability robustness and certain performance robustness, it is important to guarantee that the eigenvalues of a linear time-invariant system under parameter uncertainties remain in a specified region. Besides, time-delay is commonly encountered in various engineering systems ${ }^{(1)}$. Hence, in recent years, some researchers ${ }^{(2)-(9)}$ have investigated the robustness problem of eigenvalue-clustering inside a specified region for the following linear discrete time-delay system with parameter uncertainties described by:

$$
x(k+1)=\sum_{i=0}^{p}\left(A_{i}+\Delta A_{i}\right) x(k-i)
$$

* Received 11th August, 2003 (No. 03-5099)

** Department of Mechanical Engineering, National Kaohsiung University of Applied Sciences, 415 ChienKung Road, Kaohsiung 807, Taiwan, Republic of China.

*** Department of Mechanical and Automation Engineering, National Kaohsiung First University of Science and Technology, 1 University Road, Yenchao, Kaohsiung 824, Taiwan, Republic of China.

E-mail: choujh@ccms.nkfust.edu.tw where $A_{i} \in R^{n \times n}, x(k+i)$ and $x(k-i)$ are the $n \times 1$ state vectors, and $\Delta A_{i}(i=0,1,2, \ldots, p)$ denote the $n \times n$ timeinvariant parameter uncertain matrices. For the linear discrete time-delay system (1), if we set

$$
\hat{x}(k)=\left[x^{T}(k), x^{T}(k-1), x^{T}(k-2), \ldots, x^{T}(k-p)\right]^{T},(2)
$$

then the system (1) becomes the following higher order system:

$$
\hat{x}(k+1)=(\hat{A}+\Delta \hat{A}) \hat{x}(k)
$$

where

$$
\hat{A}=\left[\begin{array}{cccccc}
A_{0} & A_{1} & A_{2} & \cdots & A_{p-1} & A_{p} \\
I & 0 & 0 & \cdots & 0 & 0 \\
0 & I & 0 & \cdots & 0 & 0 \\
\cdot & \cdot & \cdot & \cdots & . & \cdot \\
. & . & . & \cdots & . & . \\
0 & 0 & 0 & \cdots & I & 0
\end{array}\right],
$$

and

$$
\Delta \hat{A}=\left[\begin{array}{cccccc}
\Delta A_{0} & \Delta A_{1} & \Delta A_{2} & \cdots & \Delta A_{p-1} & \Delta A_{p} \\
0 & 0 & 0 & \cdots & 0 & 0 \\
0 & 0 & 0 & \cdots & 0 & 0 \\
. & . & . & \cdots & . & \cdot \\
\cdot & . & . & \cdots & . & \cdot \\
0 & 0 & 0 & \cdots & 0 & 0
\end{array}\right],
$$

in which $I$ denotes the $n \times n$ identity matrix, and $\hat{A}$ and $\Delta \hat{A}$ are matrices of dimension $n(p+1) \times n(p+1)$. Many 
sufficient conditions, which are reported in the literature, for analyzing the robust eigenvalue-clustering problems of linear discrete uncertain system having no time delays can be used to investigate the linear augmented discrete system (3). However, it should be noted that, for a $\tilde{p}$ increase in the time delay $p$, the numbers in both rows and columns of matrices $\hat{A}$ and $\Delta \hat{A}$ in Eqs. (4) and (5) will increase by $n \tilde{p}$, respectively. Therefore, for large delays, the sizes of matrices $\hat{A}$ and $\Delta \hat{A}$ become high and the existing sufficient conditions for the linear augmented discrete system (3) will become troublesome. Thus, in order to get around this difficulty, all the researchers ${ }^{(2)-(9)}$ directly adopted the linear discrete time-delay system (1) instead of the linear augmented discrete system (3) to develop computationally simple conditions to analyze the robustness problem of eigenvalue-clustering inside a specified region for the linear discrete time-delay system with structured (elemental) parameter uncertainties. Note that the results proposed by Hsieh et al. ${ }^{(7),(8)}$ can be applied to analyze the robustness problem of eigenvalue-clustering inside a specified region which may be allowed to be any shape, whereas the results given by Wang and Wang ${ }^{(2),(3)}$, Trinh and Aldeen ${ }^{(4)}$, Shyu and Liaw ${ }^{(5)}$, Chang ${ }^{(6)}$, and Chen et al. ${ }^{(9)}$ can only be applied to analyze the robustness problem of eigenvalueclustering within a specified circular region. For the case of eigenvalue-clustering in a circular region, the sufficient condition proposed by Hsieh et al. ${ }^{(7),(8)}$ is shown to be less conservative than those proposed by Wang and Wang ${ }^{(2),(3)}$, Trinh and Aldeen ${ }^{(4)}$, Shyu and Liaw ${ }^{(5)}$, and Chang ${ }^{(6)}$; besides, it can be seen that the sufficient condition proposed by Hsieh et al. ${ }^{(7),(8)}$ is the generalized version of the results given by Chen et al. ${ }^{(9)}$

On the other hand, it is well known that an approximate system model is always used in practice and sometimes the approximation error should be covered by introducing both structured (elemental) and unstructured (norm-bounded) parameter uncertainties in control system analysis and design ${ }^{(10)}$. That is, it is not unusual that at times we have to deal with a system simultaneously consisting of two parts: one part has only the structured parameter uncertainties, and the other part has the unstructured parameter uncertainties. But, to the authors' best knowledge, the problem of regional eigenvalue-clustering robustness analysis for linear discrete time-delay systems simultaneously with both structured (elemental) and unstructured (norm-bounded) parameter uncertainties has not been discussed in the literature. Therefore, the purpose of this paper is to study the robustness problem of regional eigenvalue-clustering for the linear discrete timedelay system (1) simultaneously with both structured (elemental) and unstructured (norm-bounded) parameter uncertainties, where the specified region may be allowed to be any shape. The main results are presented in section 2 . Three examples are given in section 3 for illustration. For the case of only considering structured (elemental) parameter uncertainties, a comparison of conservatism between the criteria, which are, respectively, proposed in this paper and by Hsieh et al. ${ }^{(7),(8)}$, is also given in this section. Finally, section 4 offers some conclusions.

\section{Regional Eigenvalue-Clustering Robustness}

Consider a linear discrete time-delay system with both structured (elemental) and unstructured (normbounded) parameter uncertainties described as Eq. (1), in which the $n \times n$ time-invariant parameter uncertain matrices $\Delta A_{i}(i=0,1,2, \ldots, p)$ include both structured and unstructured parameter uncertainties and are of the form

$$
\Delta A_{i}=\sum_{j=1}^{m} \varepsilon_{i j} H_{i j}+F_{i},
$$

where $H_{i j}(i=0,1,2, \ldots, p$ and $j=1,2, \ldots, m)$ are given constant matrices, and $\varepsilon_{i j}(i=0,1,2, \ldots, p$ and $j=1,2, \ldots, m)$ are uncertain parameters; and the unstructured uncertain $n \times n$ matrix $F_{i}(i=0,1,2, \ldots, p)$ are assumed to be bounded, i.e.,

$$
\left\|F_{i}\right\| \leq \gamma_{i},
$$

where $\gamma_{i}(i=0,1,2, \ldots, p)$ are non-negative real constant numbers, and $\|\bullet\|$ denotes any matrix norm.

In this paper, it is assumed that all the eigenvalues of the nominal discrete time-delay system

$$
x(k+1)=\sum_{i=0}^{p} A_{i} x(k-i),
$$

are located within a specified region $D$. Due to inevitable uncertainties, the nominal system (8) is perturbed into the uncertain system (1). Our problem is to determine the condition such that the uncertain system (1) has all its eigenvalues retained inside the same specified region as the nominal system (8) does.

Before we analyze the regional eigenvalue-clustering robustness of the linear uncertain discrete time-delay system (1), the following lemmas need to be introduced first.

\section{Lemma $\mathbf{1}^{(7)}$ :}

If all the eigenvalues of the nominal system (8) lie inside a specified region $D$ of the $z$-plane, then

$$
\operatorname{det}\left(z I-\sum_{i=0}^{p} z^{-i} A_{i}\right) \neq 0, \text { for } z \in Q,
$$

where $Q$ denotes the boundary of the specified region $D$.

\section{Lemma $2^{(7)}$ :}

All the eigenvalues of the uncertain system (1) lie inside the specified region $D$, if the nominal system (8) has all its eigenvalues located within a specified region $D$, and if

$$
\operatorname{det}(z I-A(\alpha)) \neq 0, \quad \text { for } z \in Q \text { and } \alpha \in[0,1],
$$

where $Q$ denotes the boundary of the specified region $D$, $A(\alpha)=A+\alpha \Delta A$ is defined as a matrix of real functions 
continuous in $\alpha$, for all $\alpha$ in [0,1], and $A(\alpha)$ satisfies $A(0)=A$ and $A(1)=A+\Delta A$, in which

$$
A=\sum_{i=0}^{p} z^{-i} A_{i}
$$

and

$$
\Delta A=\sum_{i=0}^{p} z^{-i} \Delta A_{i}
$$

\section{Lemma $3^{(11)}$ :}

The matrix measures of the matrices $W$ and $V, \mu(W)$ and $\mu(V)$, are well defined for any norm and have the following properties:

(i ) $\mu( \pm I)= \pm 1$, for the identity matrix $I$;

(ii ) $-\|W\| \leq-\mu(-W) \leq \operatorname{Re}(\lambda(W)) \leq \mu(W) \leq\|W\|$, for any norm $\|\bullet\|$ and any matrix $W \in C^{n \times n}$;

(iii) $\mu(W+V) \leq \mu(W)+\mu(V)$, for any two matrices $W, V \in C^{n \times n}$

(iv) $\mu(\gamma W)=\gamma \mu(W)$, for any matrix $W \in C^{n \times n}$ and any non-negative real number $\gamma$; where $\lambda(W)$ denotes any eigenvalue of $W$, and $\operatorname{Re}(\lambda(W))$ denotes the real part of $\lambda(W)$.

\section{Lemma $4^{(12)}$.}

For any $\gamma<0$ and any matrix $W \in C^{n \times n}, \mu(\gamma W)=$ $-\gamma \mu(-W)$.

With the preceding lemmas, now we can analyze the robustness of eigenvalue-clustering in a specified region for the uncertain discrete time-delay system (1). In what follows, we present a new sufficient criterion for ensuring that the uncertain discrete time-delay system (1) has all its eigenvalues retained inside the same specified region as the nominal system (8) does.

\section{Theorem:}

Assume that the nominal system (8) has all its eigenvalues located inside a specified region $D$. The uncertain system (1) has all its eigenvalues retained inside the same specified region $D$ as the nominal system (8) does, if the following condition is satisfied:

$$
\sum_{i=0}^{p} \sum_{j=1}^{m} \varepsilon_{i j} \phi_{i j}+\sum_{i=0}^{p} \gamma_{i} \varphi<1,
$$

where

$$
\phi_{i j}= \begin{cases}\sup _{z} \mu\left(\left(z I-\sum_{i=0}^{p} z^{-i} A_{i}\right)^{-1} z^{-i} H_{i j}\right), & \text { for } \varepsilon_{i j} \geq 0 ; \\ -\sup _{z} \mu\left(-\left(z I-\sum_{i=0}^{p} z^{-i} A_{i}\right)^{-1} z^{-i} H_{i j}\right), & \text { for } \varepsilon_{i j}<0\end{cases}
$$

and

$$
\varphi=\sup _{z}\left\|z^{-i}\left(z I-\sum_{i=0}^{p} z^{-i} A_{i}\right)^{-1}\right\|,
$$

in which $z \in Q$ and $Q$ denotes the boundary of the specified region $D$.

\section{Proof:}

Since all the eigenvalues of the nominal system (8) lie inside the specified region $D$, then, from Lemma 1 , we have

$$
\operatorname{det}\left(z I-\sum_{i=0}^{p} z^{-i} A_{i}\right) \neq 0, \quad \text { for } \quad z \in Q .
$$

From Eqs. (6), (7) and (9), and using the properties in Lemmas 3 and 4 , we can get

$$
\begin{aligned}
& \operatorname{Re}\left(\lambda\left(\sum_{i=0}^{p}\left(z I-\sum_{i=0}^{p} z^{-i} A_{i}\right)^{-1} z^{-i} \Delta A_{i}\right)\right) \\
& \leq \mu\left(\sum_{i=0}^{p} \sum_{j=1}^{m} \varepsilon_{i j}\left(z I-\sum_{i=0}^{p} z^{-i} A_{i}\right)^{-1} z^{-i} H_{i j}\right. \\
&\left.+\sum_{i=0}^{p}\left(z I-\sum_{i=0}^{p} z^{-i} A_{i}\right)^{-1} z^{-i} F_{i}\right) \\
& \leq \sum_{i=0}^{p} \sum_{j=1}^{m} \mu\left(\varepsilon_{i j}\left(z I-\sum_{i=0}^{p} z^{-i} A_{i}\right)^{-1} z^{-i} H_{i j}\right) \\
&+\sum_{i=0}^{p}\left\|\left(z I-\sum_{i=0}^{p} z^{-i} A_{i}\right)^{-1} z^{-i} F_{i}\right\| \\
& \leq \sum_{i=0}^{p} \sum_{j=1}^{m} \mu\left(\varepsilon_{i j}\left(z I-\sum_{i=0}^{p} z^{-i} A_{i}\right)^{-1} z^{-i} H_{i j}\right) \\
&+\sum_{i=0}^{p} \gamma_{i}\left\|z^{-i}\left(z I-\sum_{i=0}^{p} z^{-i} A_{i}\right)^{-1}\right\| \\
& \leq \sum_{i=0}^{p} \sum_{j=1}^{m} \varepsilon_{i j} \phi_{i j}+\sum_{i=0}^{p} \gamma_{i} \varphi \\
&<1, \quad \text { for } \quad z \in Q .
\end{aligned}
$$

Thus we have $\operatorname{Re}\left(\lambda\left(\sum_{i=0}^{p}\left(z I-\sum_{i=0}^{p} z^{-i} A_{i}\right)^{-1} z^{-i} \Delta A_{i}\right)\right)<1 / \alpha$, for $\alpha \in[0,1]$ and $z \in Q$. This implies that $\lambda\left(\sum_{i=0}^{p}\left(z I-\sum_{i=0}^{p} z^{-i} A_{i}\right)^{-1} z^{-i} \Delta A_{i}\right) \neq 1 / \alpha$, for $\alpha \in[0,1]$ and $z \in Q$. That is,

$$
\begin{aligned}
& \operatorname{det}\left(I-\alpha \sum_{i=0}^{p}\left(z I-\sum_{i=0}^{p} z^{-i} A_{i}\right)^{-1} z^{-i} \Delta A_{i}\right) \neq 0, \\
& \text { for } z \in Q \text { and } \alpha \in[0,1] .
\end{aligned}
$$

Then, from Eqs. (10) and (12), we have

$$
\begin{aligned}
& \operatorname{det}(z I-A(\alpha))=\operatorname{det}\left(z I-\sum_{i=0}^{p} z^{-i}\left(A_{i}+\alpha \Delta A_{i}\right)\right) \\
& =\operatorname{det}\left(z I-\sum_{i=0}^{p} z^{-i} A_{i}\right) \\
& \cdot \operatorname{det}\left(I-\alpha \sum_{i=0}^{p}\left(z I-\sum_{i=0}^{p} z^{-i} A_{i}\right)^{-1} z^{-i} \Delta A_{i}\right) \neq 0,
\end{aligned}
$$

for $z \in Q$ and $\alpha \in[0,1]$. Hence, from the results mentioned above and Lemma 2, we obtain that all the eigenvalues of the uncertain discrete time-delay system (1) lie inside the specified region $D$. That is, if the inequality in Eq. (9) is satisfied, the uncertain discrete time-delay system (1) has 
all its eigenvalues retained inside the specified region $D$. Thus the proof is completed.

Q.E.D.

Remark 1: If $Q$ is chosen as the unit circle and $D$ as the inside-unit-circle disc, the sufficient condition (9) becomes the robust stability criterion for the linear uncertain discrete time-delay systems.

Remark 2: If the linear uncertain system in (1) only subjects to structured (elemental) parameter uncertainties, the parameter perturbation matrix $\Delta A_{i}(i=0,1,2, \ldots, p)$ becomes

$$
\Delta A_{i}=\sum_{j=1}^{m} \varepsilon_{i j} H_{i j} .
$$

Thus, the sufficient condition (9) becomes

$$
\sum_{i=0}^{p} \sum_{j=1}^{m} \varepsilon_{i j} \phi_{i j}<1 .
$$

The sufficient condition (15) is an asymmetric sufficient condition due to that the corresponding eigenvalueclustering region on $\varepsilon_{i j}(i=0,1,2, \ldots, p$ and $j=1,2, \ldots, m)$, which is obtained by using (15), in the parameter space is not necessarily symmetric with respect to the origin of the parameter space. It will be shown later in Examples 1 and 2 given below that the asymmetric sufficient condition (15) gives less conservative results than the symmetric sufficient condition of Hsieh et al. ${ }^{(7),(8)}$, and the reason why the proposed asymmetric sufficient condition (15) is less conservative will be also explained in Remark 4 given below.

Remark 3: Here it is shown how the above results can be used to solve more complicated problems in regional eigenvalue-clustering robustness of the uncertain discrete time-delay systems. Consider the following problem: given the uncertain discrete time-delay system

$$
x(k+1)=\sum_{i=0}^{p}\left(A_{i}+\Delta \bar{A}_{i}(\bar{r})\right) x(k-i)
$$

with

$$
\Delta \bar{A}_{i}(\bar{r})=\sum_{j=1}^{m} \varepsilon_{i j}(\bar{r}) H_{i j}+F_{i}
$$

where $\varepsilon_{i j}(\bar{r})(i=0,1,2, \ldots, p$ and $j=1,2, \ldots, m)$ are given continuous functions of $\bar{r} \in R$, determine the region $S$ such that for $\bar{r} \in S$ and $\gamma_{i} \in S$, the uncertain discrete time-delay system (16) has all its eigenvalues retained inside the same specified region as the nominal system (8) does. Note that here the uncertain parameters are functions of one parameter $\bar{r}$. A similar approach can be taken when they depend on more than one parameter, however, this will not be discussed in this remark. It can be easily shown that for the more complicated uncertain matrix $\Delta \bar{A}_{i}(\bar{r})$ in (17), the proposed-above theorem still holds, and the corresponding inequality is, in this case,

$$
\sum_{i=0}^{p} \sum_{j=1}^{m} \varepsilon_{i j}(\bar{r}) \phi_{i j}+\sum_{i=0}^{p} \gamma_{i} \varphi<1
$$

The inequality in (18) serves as a starting point in the robust eigenvalue-clustering analysis of the uncertain discrete time-delay system (16). It is significant because it enables us to study the effects of both $\bar{r}$ and $\gamma_{i}$ $(i=0,1,2, \ldots, p)$ on eigenvalue-clustering of the uncertain discrete time-delay system (16). There are two possible methods to obtain the region $S$. One is an analytical method by which the bounds for $\bar{r}$ and $\gamma_{i}$ are explicitly derived from (18). However, this is not always possible due to the arbitrariness of the functions $\Delta \bar{A}_{i}(\bar{r})$ and $\varepsilon_{i j}(\bar{r})$. The other method is a graphical approach, where, with the help of computer software packages such as Matlab, we can easily plot $f\left(\bar{r}, \gamma_{i}\right)=\sum_{i=0}^{p} \sum_{j=1}^{m} \varepsilon_{i j}(\bar{r}) \phi_{i j}+\sum_{i=0}^{p} \gamma_{i} \varphi$ as a function of $\bar{r}$ and $\gamma_{i}(i=0,1,2, \ldots, p)$, and therefore determine the region $S$, which is the region that satisfies $f\left(\bar{r}, \gamma_{i}\right)<1$.

\section{Examples}

In this section, we give three examples for illustration.

\section{Example 1:}

Consider a linear uncertain discrete time-delay system described as

$$
\begin{aligned}
x(k+1)= & A_{0} x(k)+\varepsilon_{01} H_{01} x(k) \\
& +A_{1} x(k-1)+\varepsilon_{11} H_{11} x(k-1),
\end{aligned}
$$

with $\varepsilon_{01} \in[-16,1.5], \varepsilon_{11} \in[-32,1.2]$ and $\varepsilon_{01}, \varepsilon_{11} \in R$ where

$$
\begin{aligned}
& A_{0}=\left[\begin{array}{cc}
0.68 & 1 \\
0.5 & 0.42
\end{array}\right], \quad A_{1}=\left[\begin{array}{cc}
0.03 & 0.06 \\
0.01 & -0.06
\end{array}\right], \\
& H_{01}=\left[\begin{array}{cc}
0.06 & 0.04 \\
0.02 & -0.04
\end{array}\right], \quad \text { and } H_{11}=\left[\begin{array}{cc}
-0.03 & 0.01 \\
-0.01 & -0.03
\end{array}\right] .
\end{aligned}
$$

The eigenvalues of the nominal system, $x(k+1)=$ $A_{0} x(k)+A_{1} x(k-1)$, are $0.8778,-0.0344$ and $0.1283 \pm$ $\tilde{j} 0.2509$ which are located inside a specified circular region $D$ centered at $0.2+\tilde{j} 0$ with radius 0.8 , where $\tilde{j}=\sqrt{-1}$.

If we apply the sufficient condition of Hsieh et al. ${ }^{(7),(8)}$, we have, for $\omega=\exp (\tilde{j} \theta), \theta \in[0,2 \pi], \alpha=0.2$ and $\beta=0.8$,

$$
\begin{aligned}
& r\left[\beta^{-1} \sum_{i=0}^{1} \sum_{j=1}^{1}\left|\varepsilon_{i j}\right|\left|(\alpha+\beta \omega)^{-1}\right|\right. \\
& \left.\cdot\left|\left(\omega I-\beta^{-1}\left(\sum_{i=0}^{1}(\alpha+\beta \omega)^{-i} A_{i}-\alpha I\right)\right)^{-1}\right|\left|H_{i j}\right|\right] \leq 20.835 \nless 1,
\end{aligned}
$$

so we can not reach any conclusion for guaranteeing the robust eigenvalue-clustering inside the specified circular region $D$. That is, the sufficient condition of Hsieh et al. ${ }^{(7),(8)}$ cannot be applied in this example. If we use the the sufficient condition (15) with the 2-norm-based matrix measure, we can get

( i ) $\varepsilon_{01} \phi_{01}+\varepsilon_{11} \phi_{11} \leq 0.9923<1$, for $\varepsilon_{01} \in[0,1.5]$ and $\varepsilon_{11} \in[0,1.2]$;

(ii ) $\varepsilon_{01} \phi_{01}+\varepsilon_{11} \phi_{11} \leq 0.9980<1$, for $\varepsilon_{01} \in[0,1.5]$ and $\varepsilon_{11} \in[-32,0]$; 
(iii) $\varepsilon_{01} \phi_{01}+\varepsilon_{11} \phi_{11} \leq 0.9664<1$, for $\varepsilon_{01} \in[-16,0]$ and $\varepsilon_{11} \in[-32,0]$;

(iv) $\varepsilon_{01} \phi_{01}+\varepsilon_{11} \phi_{11} \leq 0.9607<1$, for $\varepsilon_{01} \in[-16,0]$ and $\varepsilon_{11} \in[0,1.2]$.

Thus, the sufficient condition (15) is satisfied. So, according to the sufficient condition (15), we can conclude that the linear uncertain discrete time-delay system (19) has all its eigenvalues retained inside the same specified circular region $D$.

\section{Example 2:}

Consider the linear uncertain discrete time-delay system described as

$$
\begin{aligned}
x(k+1)= & A_{0} x(k)+\varepsilon_{01} H_{01} x(k) \\
& +A_{1} x(k-1)+\varepsilon_{11} H_{11} x(k-1),
\end{aligned}
$$

with $\varepsilon_{01} \in[-7.8,1.8], \varepsilon_{11} \in[-2.9,4.4]$ and $\varepsilon_{01}, \varepsilon_{11} \in R$ where

$$
\begin{aligned}
& A_{0}=\left[\begin{array}{cc}
-0.4 & 1 \\
0 & -0.35
\end{array}\right], \quad A_{1}=\left[\begin{array}{cc}
0.2 & 0.2 \\
0.01 & 0.25
\end{array}\right], \\
& H_{01}=\left[\begin{array}{ll}
0.12 & 0.3 \\
0.03 & 0.1
\end{array}\right], \quad \text { and } H_{11}=\left[\begin{array}{ll}
0.01 & 0.01 \\
0.01 & 0.03
\end{array}\right] .
\end{aligned}
$$

The eigenvalues of the nominal system, $x(k+1)=$ $A_{0} x(k)+A_{1} x(k-1)$, are $0.2445,0.4$ and $-0.6972 \pm$ $\tilde{j} 0.069$ which are located inside a specified ring region $R(-0.1,0.2,0.1)$, where the ring region $R(-0.1,0.2,0.1)$ is the closure of the intersection of the exterior of the circle $C(-0.1,0.2)$ centered at $-0.1+\tilde{j} 0$ with radius 0.2 and the interior of the circle $C(0,1)$ centered at $0+\tilde{j} 0$ with radius 1 .

If we use the sufficient condition given by Hsieh et al. ${ }^{(7),(8)}$, we can get, for $\omega=\exp (\tilde{j} \theta), \theta \in[0,2 \pi]$, $\alpha_{1}=-0.1$ and $\beta_{1}=0.2, \alpha_{2}=0$ and $\beta_{2}=1$,

$$
\begin{aligned}
& r\left[\beta_{s}^{-1} \sum_{i=0}^{1} \sum_{j=1}^{1}\left|\varepsilon_{i j}\right|\left|\left(\alpha_{s}+\beta_{s} \omega\right)^{-1}\right|\right. \\
& \left.\cdot\left|\left(\omega I-\beta_{s}^{-1}\left(\sum_{i=0}^{1}\left(\alpha_{s}+\beta_{s} \omega\right)^{-i} A_{i}-\alpha_{s} I\right)\right)^{-1}\right|\left|H_{i j}\right|\right] \\
& \leq 2.8896 \nless 1, \text { for } s=1 ;
\end{aligned}
$$

and

$$
\begin{aligned}
& r\left[\beta_{s}^{-1} \sum_{i=0}^{1} \sum_{j=1}^{1}\left|\varepsilon_{i j}\right|\left|\left(\alpha_{s}+\beta_{s} \omega\right)^{-1}\right|\right. \\
& \left.\quad \cdot\left|\left(\omega I-\beta_{s}^{-1}\left(\sum_{i=0}^{1}\left(\alpha_{s}+\beta_{s} \omega\right)^{-i} A_{i}-\alpha_{s} I\right)\right)^{-1}\right|\left|H_{i j}\right|\right] \\
& \leq 5.7542 \nless 1, \text { for } s=2 .
\end{aligned}
$$

Therefore, we can not reach any conclusion for guaranteeing the robust eigenvalue-clustering inside the specified ring region $R(-0.1,0.2,0.1)$. That is, the sufficient condition given by Hsieh et al. ${ }^{(7),(8)}$ cannot be applied in this example. If we use the sufficient condition (15) with the 2-norm-based matrix measure, we can get

( i ) $\varepsilon_{01} \phi_{01}+\varepsilon_{11} \phi_{11} \leq 0.9964<1$, for $\varepsilon_{01} \in[0,1.8]$ and $\varepsilon_{11} \in[0,4.4]$; (ii ) $\varepsilon_{01} \phi_{01}+\varepsilon_{11} \phi_{11} \leq 0.9962<1$, for $\varepsilon_{01} \in[0,1.8]$ and $\varepsilon_{11} \in[-2.9,0]$;

(iii) $\varepsilon_{01} \phi_{01}+\varepsilon_{11} \phi_{11} \leq 0.9054<1$, for $\varepsilon_{01} \in[-7.8,0]$ and $\varepsilon_{11} \in[-2.9,0]$;

(iv) $\varepsilon_{01} \phi_{01}+\varepsilon_{11} \phi_{11} \leq 0.9936<1$, for $\varepsilon_{01} \in[-7.8,0]$ and $\varepsilon_{11} \in[0,4.4]$.

Therefore, the sufficient condition (15) is satisfied. According to the sufficient condition (15), we can conclude that the linear uncertain discrete time-delay system (20) has all its eigenvalues retained inside the same specified ring region $R(-0.1,0.2,0.1)$.

Remark 4: From Examples 1 and 2, we can see that the proposed asymmetric sufficient condition (15) may obtain less conservative results than the symmetric sufficient condition given by Hsieh et al. ${ }^{(7),(8)}$ The reason why the proposed asymmetric sufficient condition (15) is less conservative is that it takes the directional information into consideration. This can be explained by the fact that as a parameter varies in different directions, it affects the system's eigenvalues differently. This can be easily shown using, for example, the root-locus technique, where it is well known that, for different signs of the parameter, the root locus is completely different; that is, the effect of a single parameter $\varepsilon$ in $A$ on the eigenvalues of the nominal system (8) can be completely different for the same $|\varepsilon|$ and opposite sign. Therefore, any sufficient conditions, that ignore the signs and thus could be called symmetric sufficient conditions, may obtain more conservative results in typical cases.

\section{Example 3:}

Consider the linear uncertain time-delay system described as

$$
\begin{aligned}
& x(k+1)=A_{0} x(k)+\varepsilon_{01} H_{01} x(k)+F_{0} x(k) \\
& \quad+A_{1} x(k-1)+\varepsilon_{11} H_{11} x(k-1)+F_{1} x(k-1),
\end{aligned}
$$

with $\left\|F_{i}\right\| \leq \gamma_{i}=\gamma(i=0,1)$, where $\gamma \geq 0$, and the matrices $A_{0}, A_{1}, H_{01}$ and $H_{11}$ are the same as those given in Example 1.

Using the proposed sufficient condition (9) with the 2-norm-based matrix measure and the spectral norm, we can get that, if $\varepsilon_{01}, \varepsilon_{11}$ and $\gamma$ satisfy the following conditions:

( i ) $0.5527 \varepsilon_{01}+0.1361 \varepsilon_{11}+16.823 \gamma<1$, for $\varepsilon_{01} \geq 0$ and $\varepsilon_{11} \geq 0$;

(ii ) $-0.0498 \varepsilon_{01}+0.1361 \varepsilon_{11}+16.823 \gamma<1$, for $\varepsilon_{01}<0$ and $\varepsilon_{11} \geq 0$;

(iii) $-0.0498 \varepsilon_{01}-0.0053 \varepsilon_{11}+16.823 \gamma<1$, for $\varepsilon_{01}<0$ and $\varepsilon_{11}<0$;

(iv) $0.5527 \varepsilon_{01}-0.0053 \varepsilon_{11}+16.823 \gamma<1$, for $\varepsilon_{01} \geq 0$ and $\varepsilon_{11}<0$;

the linear uncertain discrete time-delay system (21) has all its eigenvalues retained inside the same specified region $D$ as the nominal system, $x(k+1)=A_{0} x(k)+A_{1} x(k-1)$, does, 
where the region $D$ is given in Example 1.

\section{Conclusions}

A new sufficient condition has been proposed to ensure that the linear discrete time-delay system with both structured (elemental) and unstructured (norm-bounded) parameter uncertainties has all its eigenvalues retained in the same specified region as the linear nominal discrete time-delay system does. No restriction is imposed on the shapes of the specified region. In the proposed theorem, the sufficient condition depends on the boundary of the specified region. This is not restrictive but necessary, because there is no constraint imposed on the shape of the specified region in our theorem. When all the eigenvalues are just required to locate inside the unit circle, the proposed criterion will become the stability robustness criterion. For the case that the linear discrete time-delay system only subjects to structured parameter uncertainties, by two illustrative examples, the presented sufficient condition is shown to be less conservative than that proposed by Hsieh et al. ${ }^{(7),(8)}$ The reason why the proposed sufficient condition is less conservative has been explained in Remark 4. In Remark 3, the result in the proposed theorem has also been extended to the eigenvalue-clustering analysis of linear discrete time-delay systems with both linearly unstructured uncertainties and nonlinearly dependent parameter uncertainties.

\section{Acknowledgement}

This work is supported by the National Science Council, Taiwan, Republic of China, under grant number NSC92-2213-E151-006.

\section{References}

(1) Chu, J., A Time-Delay Control Algorithm for Discrete Systems and Its Applications to an Industrial Electric Heater, Control Theory and Applications, Vol.12 (1995), pp.70-75.

( 2 ) Wang, R.J. and Wang, W.J., D-Stability Analysis of
Perturbed Discrete-Time Systems with Multiple TimeDelays, J. of Control Systems and Technology, Vol.2 (1994), pp.59-64.

( 3 ) Wang, R.J. and Wang, W.J., Disk Stability and Robustness for Discrete-Time Systems with Multiple TimeDelays, Control-Theory and Advanced Technology, Vol.10 (1995), pp.1505-1513.

(4) Trinh, H. and Aldeen, M., D-Stability Analysis of Discrete-Delay Perturbed Systems, Int. J. of Control, Vol.61 (1995), pp.493-505.

( 5 ) Shyu, K.K. and Liaw, K.Y., D-Stability Analysis for Discrete Delay Systems, J. of the Chinese Institute of Engineers, Vol.18 (1995), pp.575-581.

( 6 ) Chang, Y.H., Robust D-Stability Analysis for DiscreteTime Delay Systems via Stability Radii, J. of Control Systems and Technology, Vol.5 (1997), pp.263-276.

( 7 ) Hsieh, C.H., Chou, J.H. and Wu, Y.J., Robust Regional Eigenvalue-Clustering Analysis for Linear Perturbed Discrete Time-Delay Systems, J. of the Chinese Institute of Engineers, Vol.23 (2000), pp.125-130.

( 8 ) Hsieh, C.H., Chou, J.H. and Wu, Y.J., Robustness of Eigenvalue-Clustering in a Ring Region for Linear Perturbed Discrete Time-Delay Systems, IEICE Trans. on Fundamentals of Electronics, Communications and Computer Sciences, Vol.E84-A (2001), pp.1557-1563.

( 9 ) Chen, C.F., Pan, S.T. and Hsieh, J.G., D-Stability Analysis for a Class of Uncertain Discrete Systems with Multiple Time Delays, JSME Int. J., Ser. C, Vol.45 (2002), pp.601-605.

(10) Zhou, K. and Gu, G., Robust Stability of Multivariable Systems with Both Real Parametric and Norm Bounded Uuncertainties, IEEE Trans. on Automatic Control, Vol.37 (1992), pp.1533-1537.

(11) Desoer, C.A. and Vidyasagar, M., Feedback Systems: Input-Output Properties, (1975), Academic Press, New York.

(12) Chen, S.H., Chou, J.H. and Fong, I.K., Robust Rank Preservation of Matrices with Both Structured and Unstructured Uncertainties and Its Applications, Proceedings of the Institution of Mechanical Engineers, Part I, J. of Systems and Control Engineering, Vol.215 (2001), pp.499-504. 